\title{
Energy absorption of xenon clusters in helium nanodroplets under strong laser pulses
}

Article

Accepted Version

Mikaberidze, A., Saalmann, U. and Rost, J. M. (2008) Energy absorption of xenon clusters in helium nanodroplets under strong laser pulses. Physical Review A, 77 (4). $041201(R)$. ISSN 1050-2947 doi:

https://doi.org/10.1103/PhysRevA.77.041201 Available at https://centaur.reading.ac.uk/91224/

It is advisable to refer to the publisher's version if you intend to cite from the work. See Guidance on citing.

Published version at: http://dx.doi.org/10.1103/PhysRevA.77.041201

To link to this article DOI: http://dx.doi.org/10.1103/PhysRevA.77.041201

Publisher: American Physical Society

All outputs in CentAUR are protected by Intellectual Property Rights law, including copyright law. Copyright and IPR is retained by the creators or other copyright holders. Terms and conditions for use of this material are defined in the End User Agreement.

www.reading.ac.uk/centaur 
Central Archive at the University of Reading

Reading's research outputs online 


\title{
Energy absorption of xenon clusters in helium nanodroplets under strong laser pulses
}

\author{
Alexey Mikaberidze, Ulf Saalmann, and Jan M. Rost \\ Max Planck Institute for the Physics of Complex Systems \\ Nöthnitzer Straße 38, 01187 Dresden, Germany
}

\begin{abstract}
Energy absorption of xenon clusters embedded in helium nanodroplets from strong femtosecond laser pulses is studied theoretically. Compared to pure clusters we find earlier and more efficient energy absorption in agreement with experiments. This effect is due to resonant absorption of the helium nanoplasma whose formation is catalyzed by the xenon core. For very short double pulses with variable delay both plasma resonances, due to the helium shell and the xenon core, are identified and the experimental conditions are given which should allow for a simultaneous observation of both of them.
\end{abstract}

PACS numbers: 36.40.Gk, 31.15.Qg, 36.40.Wa

Atomic clusters couple very efficiently to laser light pulses [1, 2, 3]. Through irradiation with a strong laser pulse, typically a nanoplasma is formed which can absorb resonantly energy if its eigenfrequency matches the laser frequency [4]. While it is generally agreed by now that collective electron oscillation resonant with the laser frequency is the most efficient way to transfer energy from the laser pulse into the cluster [4, [5, [6, 7, ,8], subtle effects continue to be discussed [9, 10, 11]. On the other hand intriguing and so far not well understood phenomena have been observed in composite clusters illuminated by strong laser pulses, such as enhancement of X-ray production in water-doped clusters [12, 13] and the earlier resonant energy absorption for clusters embedded in helium droplets [14]. Growing clusters in helium droplets has proven to be an elegant alternative to their production in supersonic beams [15, 16, 17]. In contrast to spectroscopic applications [18], where the role of the helium droplet is merely to isolate and cool the embedded species, helium embedding may significantly influence cluster dynamics when a strong laser pulse is applied [14].

Here, we investigate this influence theoretically by exposing a xenon cluster (100 atoms) embedded in a helium droplet of up to 5000 atoms to a single laser pulse. It is taken sufficiently long (100 fs) for an expansion of the cluster to roughly double its original size. We find the usual resonant absorption when the eigenfrequency of the expanding xenon cluster matches the laser frequency. However, in agreement with the experiment [14], earlier in the pulse we see another resonance which dominates the energy absorption. Therefore, higher cluster charges and energy absorption are observed in a heliumembedded cluster compared to the pure one. This suggests that the resonance earlier in the laser pulse found for helium embedded lead clusters [14] is not the same one as seen for the pure lead cluster, simply shifted to earlier times due to the helium environment. Rather, it is the resonance of the embedding helium droplet and for some reason to be clarified the resonance of the xenon core is not visible.

To understand better the energy absorption, we have used the double pulse scheme and replaced the $100 \mathrm{fs}$ pulse by two time-delayed very short pulses of $10 \mathrm{fs}$ duration, during which the cluster expansion is negligible. As we will show the cluster parameters as well as those of the laser have to be chosen very carefully to see both resonances in one experiment.

We use a classical molecular dynamics approach with tree-code techniques [1, 19] to follow the cluster dynamics. The initial positions of the xenon atoms are chosen according to the lowest energy configuration [20], while helium atoms are placed randomly so that the droplet has the density of bulk liquid helium $\left(\rho=0.02185 \AA^{-3}\right)$. As ionization mechanism we assume barrier suppression which dominates at the high laser intensities considered here [6]. This means that due to electric fields created by surrounding charges and the laser the potential barrier is bent down sufficiently to release classically a bound electron from its mother ion into the cluster environment [8]. Such electrons are called "inner-ionized" and their number equals the positive background charge $Q(t)$ created. Some of them gain enough energy to leave the cluster, they become "outer-ionized". Those, which are innerionized but remain in the cluster, we call "quasi-free". They form the nanoplasma [4] which plays a crucial role for the energy absorption in the cluster [1]. All the laser pulses used have $780 \mathrm{~nm}$ wavelength. For clarity we will restrict ourselves to a xenon cluster of 100 atoms embedded in helium droplets of 300, 500, 1000 and 5000 atoms, respectively.

First, we will discuss the energy absorption of the embedded cluster from a single laser pulse of $100 \mathrm{fs}$ duration (FWHM) with the intensity $I=3.51 \times 10^{14} \mathrm{~W} / \mathrm{cm}^{2}$. Under this laser pulse, the cluster is ionized and expands. Every helium atom is doubly ionized, while the average charge of xenon is around $11+$ per ion by the end of the pulse. The expansion is much faster for the light helium ions than for the heavier xenon ions (see Fig.11). While the cluster expands, the rate of energy absorption changes dramatically, reaching two maxima, as can be seen from Fig. 22 . The first maximum is much higher than the second one. Comparison with the pure clus- 


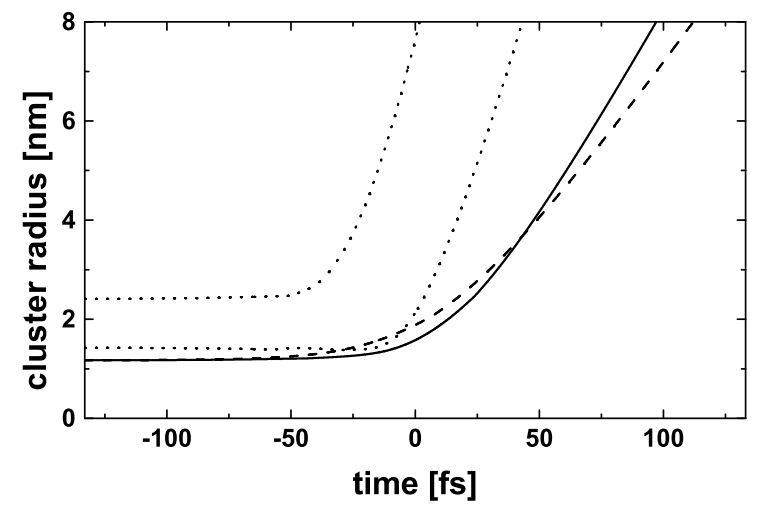

FIG. 1: Cluster radii as functions of time for the helium droplet in $\mathrm{Xe}_{100} @ \mathrm{He}_{1000}$ (dotted, inner and outer edge), for the xenon cluster in $\mathrm{Xe}_{100} @ \mathrm{He}_{1000}$ (solid), and for the bare $\mathrm{Xe}_{100}$ cluster (dashed).

ter (dashed line) indicates that the second maximum is caused by the resonant absorption due to the expanding xenon cluster: At resonance the xenon cluster has reached a critical radius $R\left(t^{\star}\right)=R_{\text {crit }}$ so that the eigenfrequency of the quasi-free electrons $\Omega(t)=\sqrt{Q(t) / R^{3}(t)}$ matches the laser frequency $\Omega\left(t^{\star}\right)=\omega$ [7]. A clearer indication for resonant absorption than the eigenfrequency is the time dependent phase shift $\phi(t)$ of the periodic center of mass (CM) motion of the quasi-free electrons with respect to the driving laser field. At resonance the phase shift is $\phi\left(t^{\star}\right)=\pi / 2$ [7]. The corresponding times $t^{\star}$ are indicated by the two vertical lines in Fig. 20 for the embedded (solid) and the pure (dashed) cluster.

Indeed, also for the first and the dominant resonance feature, the phase shift of the CM motion of all quasifree electrons passes $\pi / 2$ at the time of maximum absorption rate. Excluding the quasi-free electrons inside the xenon core of the cluster gives the same result (dotted curve in Fig.2r) which clarifies that it is the period of the electronic CM motion in the extended potential of the helium ions which coincides with the laser period. This result also explains why the early resonance is much stronger than the second one. It is simply because there are many more helium ions than xenon ones so that many more electrons participate in the resonant absorption in helium shell than in xenon core. Note, that for a larger helium droplet of 5000 atoms the second resonance is hidden by the first one and not seen in the energy absorption rate.

These findings are consistent with the earlier absorption in the embedded cluster compared to the pure one found in the experiment [14]. However, in contrast to the two resonances we have identified, only one early in the laser pulse was seen in the experiment using a pumpprobe double pulse scheme. Therefore, we will analyze

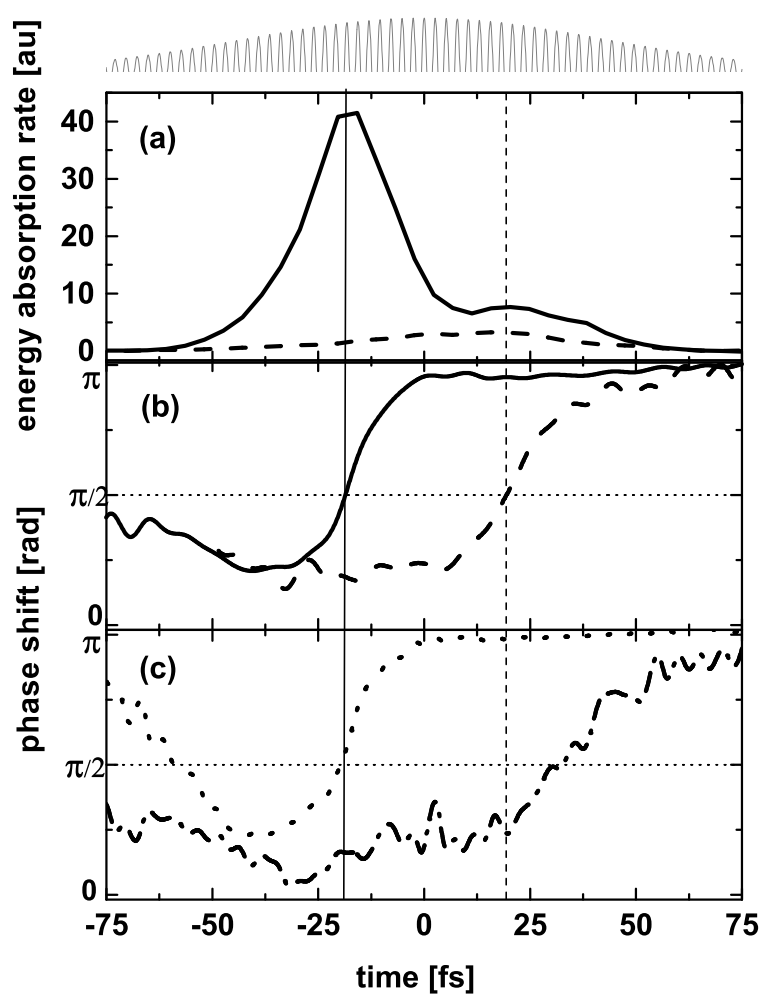

FIG. 2: Energy absorption rate (a), and phase shift of CM oscillations of the quasi-free electrons with respect to laser field (b) for $\mathrm{Xe}_{100} @ \mathrm{He}_{1000}$ (solid) and $\mathrm{Xe}_{100}$ (dashed), respectively. Phase shifts for the the embedded system Xe $\mathrm{X}_{100} @ \mathrm{He}_{1000}$ (c) from electrons in the helium shell (dotted) and in the xenon core (dash-dotted) only. The laser pulse with a peak intensity of $I=3.51 \times 10^{14} \mathrm{~W} / \mathrm{cm}^{2}$, a duration of $100 \mathrm{fs}$ and a wavelength of $\lambda=780 \mathrm{~nm}$ is shown on top of the figure.

the effect of the double pulse with variable delay on the same system as before in the following.

We have used two identical pulses with a duration of $10 \mathrm{fs}$ (FWHM) and a delay from 10 to $250 \mathrm{fs}$. Results for two different intensities of the pulses ( $I=$ $8.8 \times 10^{14} \mathrm{~W} / \mathrm{cm}^{2}$ and $I=3.5 \times 10^{14} \mathrm{~W} / \mathrm{cm}^{2}$ ) are shown in Fig. 3. Surprisingly, for the same cluster as before $\left(\mathrm{Xe}_{100} @ \mathrm{He}_{1000}\right)$, one only sees the early resonance (Fig.33) in qualitative agreement with the experiment 14] for which we conclude that there the helium resonance was observed.

For the higher intensity (Fig. $3 \mathrm{a}$ ) the resonance peak appears to be rather asymmetric having a shoulder towards longer delays where the second resonance due to xenon should be but is apparently masked by the helium resonance. There are several ways to check this assumption: The one which can also be realized experimentally is to increase the number of xenon atoms relative to the helium atoms. We did so by reducing the number of helium atoms. Indeed, for 500 helium atoms the second 


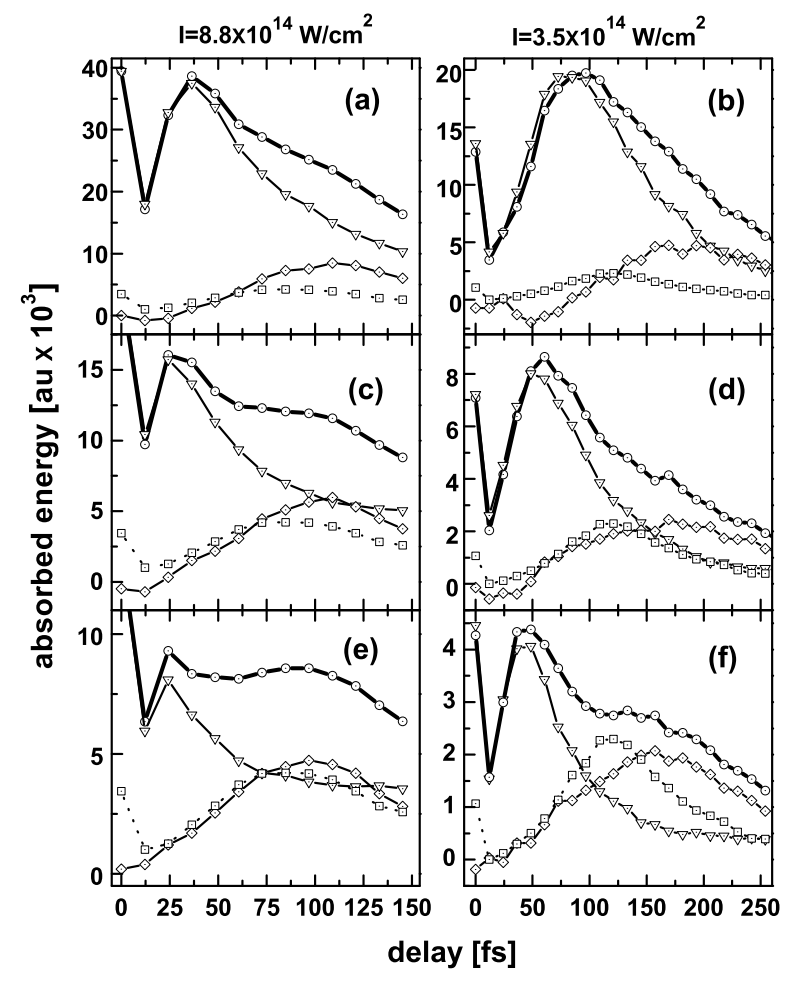

FIG. 3: Absorbed energy as a function of delay between pump and probe pulse for $\mathrm{Xe}_{100} @ \mathrm{He}_{N}$ (०), for xenon atoms fixed $(\nabla)$, for bare $\mathrm{Xe}_{100}$ clusters $(\square$, dotted line) and finally, the difference in energy absorption for helium-embedded xenon clusters with moving and fixed xenon atoms $(\diamond)$. The panels (a) and (b) are for $N=1000$ helium atoms, (c) and (d) for $N=500$ and (e) and (f) for $N=300$, respectively.

resonance appears already for the higher intensity, and for 300 helium atoms, the later resonance is visible at both laser intensities. This shows, that the parameters of the laser have to be carefully adopted for the embedded cluster under investigation in order to get the full information on energy absorption from pump-probe schemes.

To further elucidate the nature of the second smaller energy absorption peak, we have fixed the xenon atoms in space. This is, of course, only possible in a calculation. For such a situation we can exclude resonant absorption by the xenon core since the ion charge density there will be too high for matching the corresponding eigenfrequency with the laser frequency (triangles in Fig.31). Consequently, under none of the parameter combinations energy absorption for fixed xenon shows a second peak in Fig.3. To demonstrate that the xenon resonance nevertheless exists, we have constructed an artificial absorption curve (squares) by subtracting the result for fixed xenon from the full dynamical absorption (circles). Now, again for all parameter combinations, the first resonance due to the helium ions has disappeared. This indicates that it has not been affected by the presence of the fixed

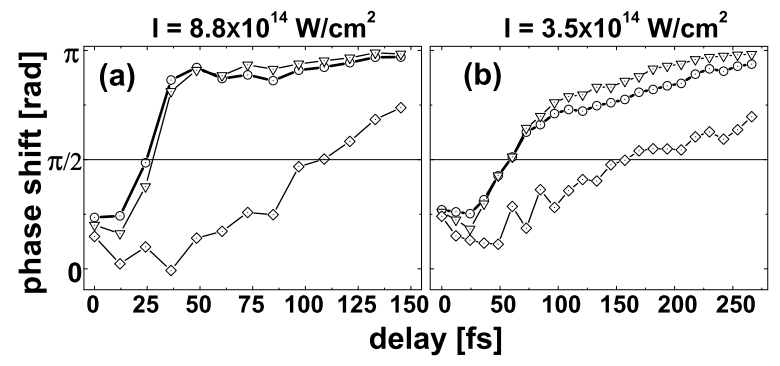

FIG. 4: Phase shift of the CM oscillation of quasi-free electrons with respect to the driving field taken at the maximum of the second pulse as a function of delay between pulses. The phase shift of all quasi-free electrons (o), of quasi-free electrons in the helium shell only $(\nabla)$ and in the xenon core $(\diamond)$ only is shown for $\mathrm{Xe}_{100} @ \mathrm{He}_{500}$.

xenon atoms and ions. On the other hand, the second resonance due to xenon clearly shows up in the difference curve, revealing that it is present but buried under the helium signal in the full dynamical energy absorption (circles) but of course by construction absent in the fixed xenon absorption (triangles). Moreover, this difference curve bares similarity with the energy absorption for a pure xenon cluster, where the general trend is that the xenon resonance is stronger and appears later in the embedded cluster. It is stronger due to the larger number of quasi-free electrons participating in the resonant absorption. They also give rise to a slightly higher ion charge $Q(t)$ of the embedded xenon cluster. Consequently, a longer expansion time is needed to reach the critical ion charge density corresponding to resonance.

We finally take a look at the phase shift of the quasifree electron CM motion versus the laser driving in Fig.4 for the case of 500 helium atoms. We have separated the quasi-free electrons contributing to the helium resonance and to the xenon one by spatial selection of the respective electrons in the cluster. The result confirms our picture: The second weaker absorption peak is due to the electrons near the xenon core. Their collective phase shift $\phi(t)$ passes $\pi / 2$ at the right time of about $100 \mathrm{fs}$ and $160 \mathrm{fs}$ for the two laser intensities, respectively.

From these results one could get the impression, that in a composite cluster of two atomic species two resonances occur related to the respective two resonance frequencies, or specifically in our case: we observe the helium resonance of the droplet almost not influenced by the xenon core. This is, however, by no means true: We have verified that without the xenon core, helium is not ionized at all for the laser intensities used. This is clear recalling that the intensities are not large enough to ionize helium by barrier suppression, while multiphoton ionization requires some 20 photons and is very unlikely. Moreover, when the laser intensity is increased so that barrier 
suppression ionization of helium becomes possible, our calculations show that resonant absorption is of minor importance (less than $20 \%$ of the total absorbed energy) in contrast to a bare cluster. The reason is that double ionization follows single ionization immediately, as soon the laser intensity is enough for single ionization of helium, due to strong fields that build up in the cluster environment. After that, there is only a small number of quasi-free electrons left in the cluster, which become outer ionized as the cluster approaches the plasma resonance. On the other hand, a xenon core (or similar) is more easily ionized and first drives the electrons, removed from helium, to the center of the cluster instead of loosing them. Thus, it is only the composite cluster (xenon cluster in a helium droplet) that exhibits the earlier resonance leading to very strong energy absorption. Neither the helium nor the xenon cluster by itself has this property.

To summarize, we have shown that a helium embedding strongly influences the dynamics of rare gas clusters illuminated by strong laser pulses. For both, single and double laser pulses, the helium droplet leads to the appearance of an additional plasma resonance, occurring earlier in the laser pulse. Its weight relative to the plasma resonance in the xenon cluster core depends on the size of the droplet compared to the size of xenon cluster and on the laser intensity. This finding should allow to choose the parameters such that an observation of both resonances in one double-pulse experiment can be realized. The dominant resonance in helium droplet occurs earlier because light helium ions explode faster than xenon ions. Due to this additional resonance and also due to the larger number of quasi-free electrons the helium embedding increases the energy absorption of the cluster. This indicates that helium embedding is quite dangerous if one is interested in the properties of the embedded clusters [21], since the dynamics of the helium droplet dominates the absorption properties of the composite cluster in most cases.

Furthermore, based on the results presented we conjecture that significant resonant absorption in a helium droplet is only possible in a composite cluster unless the helium droplet is very large. From the perspective of xenon, however, the helium embedding makes the absorption slightly stronger since more electrons, also from helium, are available. Finally, our results suggest that it may be well possible to obtain, e.g., very fast electrons or $\mathrm{X}$-rays dominantly of a certain wavelength by specifically choosing a certain composite cluster in combination with a suitable dual laser pulse. Studies in this direction are under way.

We thank Ionuţ Georgescu and Christian Gnodtke for helpful discussions and the International Max Planck Research School "Dynamical Processes in Atoms, Molecules and Solids" for financial support.

[1] U. Saalmann, C. Siedschlag, and J. M. Rost, J. Phys. B 39, R 39 (2006).

[2] V. P. Krainov and M. B. Smirnov, Phys. Rep. 370, 237 (2002).

[3] Molecules and clusters in intense laser fields, edited by J. Posthumus (Cambridge University Press, Cambridge, 2001).

[4] T. Ditmire, T. Donnelly, A. M. Rubenchik, R. W. Falcone, and M. D. Perry, Phys. Rev. A 53, 3379 (1996).

[5] L. Köller, M. Schumacher, J. Köhn, S. Teuber, J. Tiggesbäumker, and K. H. Meiwes-Broer, Phys. Rev. Lett. 82, 3786 (1999).

[6] I. Last and J. Jortner, Phys. Rev. A 60, 2215 (1999).

[7] U. Saalmann and J. M. Rost, Phys. Rev. Lett. 91, 223401 (2003).

[8] U. Saalmann, J. Mod. Opt. 53, 173 (2006).

[9] C. Jungreuthmayer, M. Geissler, J. Zanghellini, and T. Brabec, Phys. Rev. Lett. 92, 133401 (2004).

[10] M. Kundu and D. Bauer, Phys. Rev. Lett. 96, 123401 (2006).

[11] T. Fennel, T. Döppner, J. Passig, C. Schaal, J. Tiggesbäumker, and K.-H. Meiwes-Broer, Phys. Rev. Lett. 98, 143401 (2007).

[12] J. Jha, D. Mathur, and M. Krishnamurthy, J. Phys. B 38, L 291 (2005).

[13] J. Jha, P. Sharma, V. Nataraju, R. K. Vatsa, D. Mathur, and M. Krishnamurthy, Chem. Phys. Lett. 430, 26 (2006).

[14] T. Döppner, S. Teuber, T. Diederich, T. Fennel, P. Radcliffe, J. Tiggesbäumker, and K. H. Meiwes-Broer, Eur. Phys. J. D 24, 157 (2003).

[15] M. Lewerenz, B. Schilling, and J. P. Toennies, J. Chem. Phys. 102, 8191 (1995).

[16] T. Döppner, T. Diederich, J. Tiggesbäumker, and K. H. Meiwes-Broer, Eur. Phys. J. D 16, 13 (2001).

[17] J. Tiggesbäumker and F. Stienkemeier, Phys. Chem. Chem. Phys. 9, 4748 (2007).

[18] F. Stienkemeier and K. K. Lehmann, J. Phys. B 39, R 127 (2006).

[19] J. E. Barnes and P. Hut, Nature 324, 446 (1986).

[20] D. J. Wales, J. P. K. Doye, A. Dullweber, M. P. Hodges, F. Y. Naumkin, F. Calvo, J. Hernández-Rojas, and T. F. Middleton, The Cambridge Cluster Database, 2007, http://www-wales.ch.cam.ac.uk/CCD.html

[21] T. Döppner, T. Fennel, T. Diederich, J. Tiggesbäumker, and K. H. Meiwes-Broer, Phys. Rev. Lett. 94, 013401 (2005). 